ESJ Humanities

\title{
Campagne de Communication «Zéro Grossesse À L’école » et Problématique du Changement de Comportements Chez les Élèves en Matière de Sexualité en Côte d'Ivoire
}

\author{
Dr. Sey Henri Jö̈l \\ Enseignant-Chercheur à l'UFRICA, \\ Université Félix Houphouët-Boigny Abidjan, RCI
}

Doi:10.19044/esj.2021.v17n9p54

Submitted: 21 July 2020

Accepted: 23 March 2021

Published: 31 March 2021
Copyright 2021 Author(s)

Under Creative Commons BY-NC-ND

4.0 OPEN ACCESS

Cite As:

Sey H.J. (2021). Campagne de Communication "Zéro Grossesse À L'école » et Problématique du Changement de Comportements Chez les Élèves en Matière de Sexualité en Côte d'Ivoire. European Scientific Journal, ESJ, 17(9), 54.

https://doi.org/10.19044/esj.2021.v17n9p54

\section{Résumé}

Cet article, s'inscrivant dans le champ de la communication pour le développement, étudie la stratégie de communication mise en œuvre par le ministère ivoirien de l'éducation nationale et ses partenaires en vue d'endiguer le phénomène des grossesses en milieu scolaire. Lancée en 2014, cette campagne dénommée "Zéro grossesse à l'école » cours en ce moment dans les établissements secondaires en Côte d'Ivoire. La méthodologie appliquée procède tout d'abord à l'analyse des documents (affiches et posters géants) mis à la disposition des élèves. Ensuite, un entretien semi-directif a été organisé avec un échantillon de cent (100) élèves constitué selon la méthode d'échantillonnage de volontaires. Ces élèves proviennent de l'ensemble du pays. Les résultats obtenus montrent des insuffisances et incohérences dans les affiches et messages contenus dans la campagne «Zéro grossesse à l'école » d'une part et de l'autre, ces écueils occasionnent des incompréhensions chez les élèves, et les exposent à des comportements à risques en rapport avec le sexe.

Mots-clés : Grossesse, élève, communication sociale, changement de comportement, éducation à la sexualité 


\title{
Communication Campaign "Zero Pregnancy in School" and Problematic of Changing Behavior Among Students in Sexuality in Côte d'Ivoire
}

\author{
Dr. Sey Henri Jö̈l \\ Enseignant-Chercheur à l'UFRICA, \\ Université Félix Houphouët-Boigny Abidjan, RCI
}

\begin{abstract}
This paper, which is based on the field of communication and development, focuses on the communication strategy implemented by the Ivorian Ministry of Education and its partners with a view of confining the phenomenon of pregnancies in school. Launched in 2014, this campaign known as «Zero pregnancy at the school» is currently underway in the secondary establishments in Côte d'Ivoire. The methodology employed begins with the analysis of documents (posters and huge posters) which is first of all made available to the students. Thereafter, a semi-directive maintenance was organized with a sample of one hundred (100) students formed by using the volunteers' sampling method. These students came from across different countries. The results obtained show insufficiency and disconnectedness in posters and messages contained in the «Zero pregnancy at school» campaign on one hand, while on the other hand, these pitfalls have caused students to misunderstand and expose themselves to risks related to sex.
\end{abstract}

Keywords: Pregnancy, student, social communication, behavior change, sexuality education

\section{Introduction}

Occupant actuellement une place de choix dans l'agenda des Objectifs de Développement Durable (ODD), la santé de la reproduction des adolescents et des jeunes revêt un intérêt majeur dans le monde de la recherche sociodémographique en Afrique sub-saharienne. Les grossesses en milieu scolaire constituent un phénomène qui empêche la réalisation complète des ODD spécifiquement la scolarisation, la promotion de l'égalité des sexes et l'autonomisation des femmes.

Ce phénomène de grossesse en milieu scolaire est de plus en plus fréquent en Afrique sub-saharienne en général et particulièrement en Côte d'Ivoire. En 2013, 5076 cas de grossesses ont été dénombrés chez les adolescentes à l'école dans le pays (DMOSS). C'est le déclic qui a fait naître chez les gouvernants ivoiriens la volonté de lutter contre ce fléau. Ainsi, la 
campagne de sensibilisation dénommée «Zéro grossesse à l'école » voit-elle le jour en 2014. Pour les concepteurs, cette campagne vise la modification des comportements des élèves. Pour ce faire, des actions et activités tout azimut sont entreprises pour venir à bout de ce phénomène notamment des cours sur la reproduction sexuelle donnés aux élèves; la présentation des comportements à risques en rapport avec le sexe au travers de sketches et théâtres dans les écoles et espaces publics ; des psychologues sont affectés dans les établissements scolaires publics; des affiches de sensibilisation placardées sur l'ensemble du territoire ivoirien; etc. Cependant, en 2020 soit six années après la mise en œuvre de toutes ces actions, la campagne de lutte contre les grossesses en milieu scolaire ne porte pas de résultats probants, reluisants. Les filles qui contractent des grossesses durant leur parcours scolaire continuent d'être recensées avec acuité. La Direction des Stratégies, de la Planification et des Statistiques (DSPS) du Ministère de l'éducation nationale, de l'enseignement technique et de la formation professionnelle (MENET-FP) à travers sa Direction de la Mutualité et des œuvres sociales en milieu scolaire a enregistré au cours des cinq (5) années après la mise en œuvre de la campagne «Zéro grossesse à l'école » dans tout le pays : 5350 cas de grossesses en 2014 - 2015 ; 4875 cas de grossesses en 2015 - 2016 ; 4276 cas de grossesses en 2016 - $2017 ; 4767$ cas de grossesses en 2017 - 2018 et 4959 cas de grossesses en 2018 - 2019 et ce dans tous les ordres d'enseignement (primaire, secondaire général et technique). Ces chiffres sont certes en légère baisse comme a bien pu le révéler le ministère de l'éducation nationale, de l'enseignement technique et de la formation professionnelle ivoirien qui a annoncé lors d'un point de presse la réduction de 33,53\% des grossesses en milieu scolaire. Cependant, ce fléau demeure toutefois présent dans le système scolaire ivoirien. Pourquoi ces campagnes ne suscitent-elles pas une prise de conscience voire un changement de comportement radical chez les élèves ? Cet article se propose principalement de dresser un état critique de la campagne de communication dénommée "Zéro grossesse à l'école » et secondairement expliquer les mécanismes d'une bonne communication sociale susceptible d'initier un véritable changement de comportements chez les adolescents ou élèves. Les insuffisances contenues dans les nombreuses campagnes de sensibilisation et d'information entreprises par le ministère ivoirien de l'éducation nationale et ses partenaires sont à la base des comportements à risques des élèves en rapport avec le sexe : telle est l'hypothèse générale qui sous-tend cette étude.

\section{Méthodologie}

\subsection{Matériels}

Les élèves issus du secondaire général tout comme technique des établissements publics et privés sur l'étendue du territoire ivoirien constitue la 
cible principale de cette étude. Cependant, vu l'immensité de l'effectif des élèves de Côte d'Ivoire et de nos charges académiques, nous avons décidé d'attendre les vacances pour aller à la rencontre des élèves.

Notre enquête s'est déroulée dans le district d'Abidjan précisément dans la commune de Yopougon du mois d'Août à celui de Septembre 2019. Cette commune, porte d'entrée du grand Abidjan, accueille presque tous les élèves de la Côte d'Ivoire en période de grandes vacances.

Cette période est choisie par nombre d'établissements pour l'organisation de cours de renforcement dénommés « cours de vacances » à l'endroit des apprenants ou élèves vacanciers. Cela est, pour nous, une aubaine dans la mesure où nous avons souhaité connaître les avis de tous élèves issus des quatre coins du pays. De manière concrète, nous sommes allés à la rencontre des élèves dans trois (3) établissements abritant les « cours de vacances » à savoir SIPOREX 3; SIPOREX 4 et le collège les Phalènes 2. Toutes ces écoles sont situées dans le quartier «Toits rouges » de la commune de Yopougon. Ce quartier regorge d'établissements accueillant de nombreux élèves venant d'horizons divers durant les vacances pour des cours de mise à niveau. Ce quartier est fortement peuplé et les élèves y sont nombreux.

Dans l'optique de connaître de manière efficiente la pensée des élèves, l'enquête de type qualitatif a été privilégiée au cours de notre étude auprès d'un échantillon s'élevant à cent (100) élèves choisis selon la méthode d'échantillonnage de volontaires. Cet échantillon a été subdivisé en cinq (5) groupes de vingt (20) élèves avec une dominance féminine. Ainsi, avons-nous réuni douze (12) filles et huit (8) garçons par groupe.

L'outil principal qui a permis de recueillir les informations nécessaires à cette étude est le guide d'entretien. En outre, des images, affiches édictées par le ministère de l'éducation nationale et ses partenaires pour la sensibilisation des élèves sur les grossesses à l'école ont été utilisées. Avec ces instruments, nous avons cherché à mesurer le niveau de compréhension des affiches et messages par les élèves et à connaître leurs attitudes vis-à-vis du sexe et ses conséquences.

\subsection{Technique d'analyse et théories de référence}

Les données recueillies à l'aide d'un dictaphone ont été analysées à partir de la théorie de changement de comportement développée par Prochaska et Di Clemente (1982) et de l'approche Connaissances, Attitudes et Pratiques (C.A.P), outil stratégique permettant d'identifier, en matière d'éducation, les besoins. La théorie de changement de comportement, quant à elle, se fonde sur la psychothérapie avec des auteurs tels que Freud, Skinner, etc. Pour elle, le changement de comportement de l'individu se déroule selon différentes étapes ordonnées et chronologiques. Ces étapes sont la pré-contemplation (conscientisation), la contemplation (réévaluation personnelle), la préparation 
(engagement), l'action (gestion des renforcements, relations aidantes) et le maintien. Ces étapes constituent ce qu'ils ont nommé le modèle transthéorique.

L'approche CAP a consisté d'une part à évaluer les connaissances des élèves après avoir été exposés aux messages de sensibilisation dans le cadre du programme "Zéro grossesse à l'école » et d'autre part à analyser leurs attitudes et pratiques, c'est-à-dire « les actes réels accomplis par la personne en situation, dans son contexte » (Goutille, 2009).

\section{Résultats}

\subsection{La sexualité: connaissances et perceptions des élèves}

La répartition par sexe nous donne de constater que nous avons interviewé soixante (60) filles contre quarante (40) garçons. Ce choix s'expliquer par le fait que nous voulons connaitre les avis des jeunes filles étant donné que ce sont elles les premières victimes des grossesses précoces contractées en milieu scolaire.

L'âge minimum pour participer à l'entretien est neuf (9) ans. Ainsi, les élèves que nous avons rencontrés ont à $92 \%$ un âge compris dans la tranche 9 à 18 ans contre $08 \%$ ayant un âge qui se situe dans la tranche plus de 18 ans. Les élèves rencontrés sont en pleine adolescence. C'est une période où l'enfant enregistre des modifications et transformations sur les plans physiologique et psychologique. Selon les spécialistes en psychologie sociale, cette période traduit chez l'adolescent l'éveil des sens et de découvertes nouvelles. Du coup, les informations pour leur équilibre physique et moral notamment les connaissances liées à la sexualité ne devraient leur manquer.

Notre étude a enregistré $45 \%$ d'élèves qui fréquentent le second cycle du secondaire et $55 \%$ au premier cycle. Dans de nombreux secteurs d'activités notamment celui de la santé, le facteur déterminant dans la compréhension des messages est le niveau d'étude. Ainsi, parvenir à mieux comprendre les messages pourrait d'emblée occasionner une prise de conscience chez l'individu et provoquer la mise en œuvre du comportement souhaité ou voulu. Les élèves interrogés proviennent des localités d'Agnibilékro ; de Toumodi ; d'Agboville ; d'Abengourou ; de Bingerville ; de Yopougon ; d'Attécoubé ; de Cocody et du Plateau. L'état ivoirien ne parvient plus à contenir les enfants dans les établissements publics. Pour l'aider dans sa tâche, il fait appel au secteur privé (les établissements privés) pour éduquer les enfants que les citoyens leur confient. Ainsi, 54\% des élèves rencontrés sont issus des établissements privés et $46 \%$ sont issus des établissements publics du pays. Au cours de nos enquêtes, nous avons constaté que les actions en matière de promotion d'un comportement ou autre diffèrent d'un établissement public à un établissement privé. Dans les établissements privés, l'état joue le rôle de simple conseillé contrairement aux établissements publics où il est acteur. 
Poser des questions aux élèves sur l'objet de notre étude à savoir la réception des messages de la campagne de sensibilisation dénommée «Zéro grossesse à l'école » initiée par le ministère de l'éducation nationale, a constitué la seconde phase de notre étude. La première question que nous leur avons posée est «qu'est-ce que la sexualité? ». L'ensemble des élèves que nous avons rencontrés a donné des réponses identiques à savoir «c'est le fait qu'un homme et une femme ont des rapports sexuels ». L'analyse de cette réponse nous permet de constater que les élèves ne connaissent qu'une partie du thème « sexualité ». Cette carence a pour conséquence une méconnaissance ou une chosification de ce thème. Pour ces derniers, sexualité est égal au sexe.

La seconde question a cherché à connaitre la définition de « grossesse ». Définir ce terme n'a guère été facile pour les élèves. Ainsi, $80 \%$ affirment que "C'est quand la femme a un gros ventre et il y a là-dedans un bébé en même temps elle va à l'école. » et $20 \%$ voient en grossesse précoce «la déformation ventrale d'une femme ». Chaque élève a sa définition. Pour Louis Dubrisay et Cyrille Jeannin (1946) "la grossesse est l'état qui commence lors de la fusion du spermatozoïde avec l'ovule, et prend fin avec l'expulsion ou l'extraction du produit de conception ». En d'autres termes, la grossesse est le processus qui part de la conception de l'enfant jusqu'à la mise au monde du bébé.

À la question de savoir «As-tu des rapports sexuels ? »,65\% affirment ne pas avoir de rapports sexuels contre $35 \%$ qui pratiquent les rapports sexuels. La majorité des élèves ayant répondu par la négation est issue du premier cycle du secondaire avec un âge qui oscille entre 9 et 15 ans. Pour eux, ils ne sont pas prêts à avoir des rapports sexuels. Ce retard ou abstinence pourrait s'expliquer par l'immaturité physique de leurs organes génitaux et le regard des autres. Cela ne veut pas dire qu'ils n'ont aucune conception ou représentation du sexe. Les $35 \%$ des élèves restants ont déjà franchi le cap des rapports sexuels. Les filles et les garçons issus du second cycle de l'enseignement secondaire privé et public pratiquent presque quotidiennement le sexe. Par ailleurs, ceux-ci ont mentionné avoir des rapports sexuels protégés. Une chose que nous avons prise avec réserve dans la mesure où ces derniers sont pratiquement vulnérables face à la tentation, au plaisir et du fait aussi que l'acte sexuel se déroule dans une intimité où seuls l'homme et la femme sont présents. En fait, vue le caractère très intimiste des rapports sexuels, il nous a été difficile de croire en leurs propos.

« Comment la grossesse se contracte-t-elle? », les réponses sont toutes identiques à savoir en ayant des rapports sexuels non protégés. De cette réponse, nous retenons que le mode ou la manière de contracter une grossesse est su de tous les participants. Cependant, pourquoi assiste-t-on de façon récurrente à des grossesses chez les jeunes filles en milieu scolaire ? Cette question vise à connaître les causes réelles des grossesses à l'école. 
Nos recherches nous ont permis de découvrir des causes aussi multiples que variées dont les plus importantes sont : l'ignorance ; la sexualité clandestine ; la non ou mauvaise application des méthodes contraceptives ; le difficile accès aux méthodes contraceptives (coût élevé pour certains élèves) ; le manque d'éducation à la sexualité ; le harcèlement sexuel de jeunes filles par certains enseignants ou personnels administratifs des établissements et la pauvreté. Les campagnes de sensibilisation sur les grossesses à l'école après analyse, ne prennent pas en compte les aspirations et représentations que les jeunes filles et garçons (adolescent-e-s) ont du sexe. L'idée que l'on se fait d'une chose guide les pas, les actions de l'homme à l'endroit de celle-là. En psychologie, la notion de représentation renvoie à la représentation mentale d'un objet en associant une perception à une idée, une catégorie de faits, une image mentale, un symbole ou un modèle explicatif. Pour être plus clair sur ce sujet, représentation est le système cognitif humain, des entités dont le rôle est de conserver l'information résultant des interactions de l'individu avec le monde et de maintenir cette information sous une forme utilisable pour des objectifs comportementaux ultérieurs. Ces entités ne sont pas des objets observables.

L'objectif de la psychologie est de rendre compte de la façon dont les individus élaborent des représentations internes et en font usage en vue de réaliser une meilleure adaptation à leur environnement. L'activité sexuelle, pendant les premières années de l'adolescence, évolue parallèlement à la maturité physique du / de la jeune (garçon, fille). En effet, les changements physiques et hormonaux mettent l'adolescent en situation de s'engager dans une plus grande variété d'activités sexuelles. Le contact régulier entre pairs à l'adolescence, fait naitre une composante clairement sexuelle. Les adolescent(e)s aiment à s'admirer et à observer les premiers signes du développement pubertaire et changements les faisant accéder à l'adolescence. Ce passage suscite parfois chez l'adolescent(e) le désir de jouir des privilèges et avantages que lui offre son nouveau corps notamment la satisfaction sexuelle restée incomplète. Le contexte socioculturel joue un rôle déterminant dans la forme que la sexualité prend à l'adolescence (Rodriguez-Tomé et al., 1997). C'est dire que la signification sociale de chaque élément du changement physique doit être communiquer à l'adolescent(e) avec clarté et succès. Ainsi, la précocité ou l'activité sexuelle intense et le vagabondage sexuel chez l'adolescent(e) tirent-ils leurs origines du construit cognitif lié à la sexualité de ce(tte) dernier(ière).

$\mathrm{Au}$ regard de ce qui précède, les véritables causes occasionnant la recrudescence des grossesses précoces sont à chercher ailleurs à savoir dans le psychique de l'adolescent. Se focaliser uniquement sur l'environnement de ce dernier, c'est perdre de vue l'essentiel. 


\subsection{La campagne « Zéro grossesse à l'école » : regard critique}

Dans la région du Gontougo précisément à Bondoukou, le ministre de l'éducation nationale a lancé, le vendredi 28 février 2014, la campagne de communication "Zéro grossesse à l'école ». Nous avons la première phase qui consiste à sensibiliser les apprenants sur les dangers des grossesses à l'école ; la seconde est la campagne d'affichages de posters et la troisième phase la prise en charge des filles-élèves enceintes à l'école par les infirmiers et aux agents de santé en service dans les établissements d'enseignement secondaire et primaire publics. Ainsi, nous avons, auprès des élèves, cherché à connaitre leur niveau de compréhension des messages associés aux images auxquels ils sont exposés jour et nuit par le concours des ministères de l'éducation nationale et de la santé publique accompagnés de leurs partenaires (UNFPA, AIMAS, etc.).

L'un des objectifs majeurs de ces affiches de communication est: d'informer, éduquer et communiquer avec les élèves sur la santé sexuelle et reproductive (SSR) pour un changement de comportement. En d'autres termes, le but poursuivi par ces entités est d'amener les élèves à changer de comportements en matière de sexualité de sorte à ce qu'ils abandonnent tout ce qui pourrait mettre en péril ou impacter négativement leurs études.

Pour connaitre le niveau de compréhension que les élèves ont de ces affiches, nous leur avons posé cette première question : "As-tu déjà suivi, écouté ou vu une campagne sur Zéro grossesse à l'école ? », les élèves nous ont tous répondu par l'affirmatif. Les enquêtés ont tous été au moins une fois exposés à la campagne, ils ont déjà vu aussi des affiches les sensibilisant sur ce fléau. Par ailleurs, pour nous assurer que ces derniers ont effectivement vu et lu un message de cette campagne, nous leur avons demandé de nous citer deux (02) publicités ou de décrire des affiches ou des messages ayant retenu leur attention. $43 \%$ des interrogés sont parvenus à décrire des affiches et publicités contre $57 \%$ qui n'ont pu le faire. Ayant vu et entendu les publicités et messages, les élèves sont, cependant, dans l'incapacité de les citer, décrire lorsqu'on leur demande. Cette situation appelle quelques observations supplémentaires. Les publicités auxquelles, les $57 \%$ des élèves ont été exposées n'ont eu de cesse à donner des informations au lieu de communiquer avec la cible (les élèves). La diffusion d'un message dans le cadre d'un changement de comportement doit amener les populations à comprendre les intérêts qu'elles ont à soutenir les initiatives prônées dans lesdits messages. Cela est loin d'être le cas dans cette campagne de sensibilisation.

L'enjeu véritable du développement des messages en matière de communication pour le développement est de promouvoir des valeurs nouvelles et non de fournir des informations. Fournir des croyances, promouvoir des comportements nouveaux afin de favoriser le changement de comportement dans le sens souhaité demeurent les maîtres-mots de ce type de 
communication. Un élève qui ne parvient pas à donner une publicité ou un message sur la campagne « Zéro grossesse à l'école » qu'il a vu et/ou entendu, c'est que le message n'est pas passé, assimilé par la cible. La communication pour le développement adoptée par le ministère de l'éducation nationale et ses partenaires est bien souvent pensée dans le sens du transfert de technologie, dans le sens de la sensibilisation mettant l'accent sur la quantité d'informations à transmettre. De ce qui précède, il est clair que les approches utilisées n'invitent pas au passage à l'acte, à l'adoption et maintien du comportement adopté. Malgré le fait que les adolescent(e)s soient exposé(e)s aux messages de sensibilisation contre les grossesses à l'école, ils n'adoptent pas le comportement que les concepteurs (ministère et partenaires) promeuvent à savoir s'abstenir de tout rapport sexuel durant leurs études. En effet, les statistiques indiquent des chiffres alarmant sur le terrain : 5350 cas de grossesses en 2014 - $2015 ; 4875$ cas de grossesses en 2015 - 2016; 4276 cas de grossesses en 2016 - 2017 ; 4767 cas de grossesses en 2017 - 2018 et 4959 cas de grossesses en 2018 - 2019 (DSPS). Ainsi, le taux des grossesses en milieu scolaire connaît une fluctuation ces cinq (5) dernières années. La figure ci-après présente le niveau d'evolution des grossesses durant les années susmentionnées.

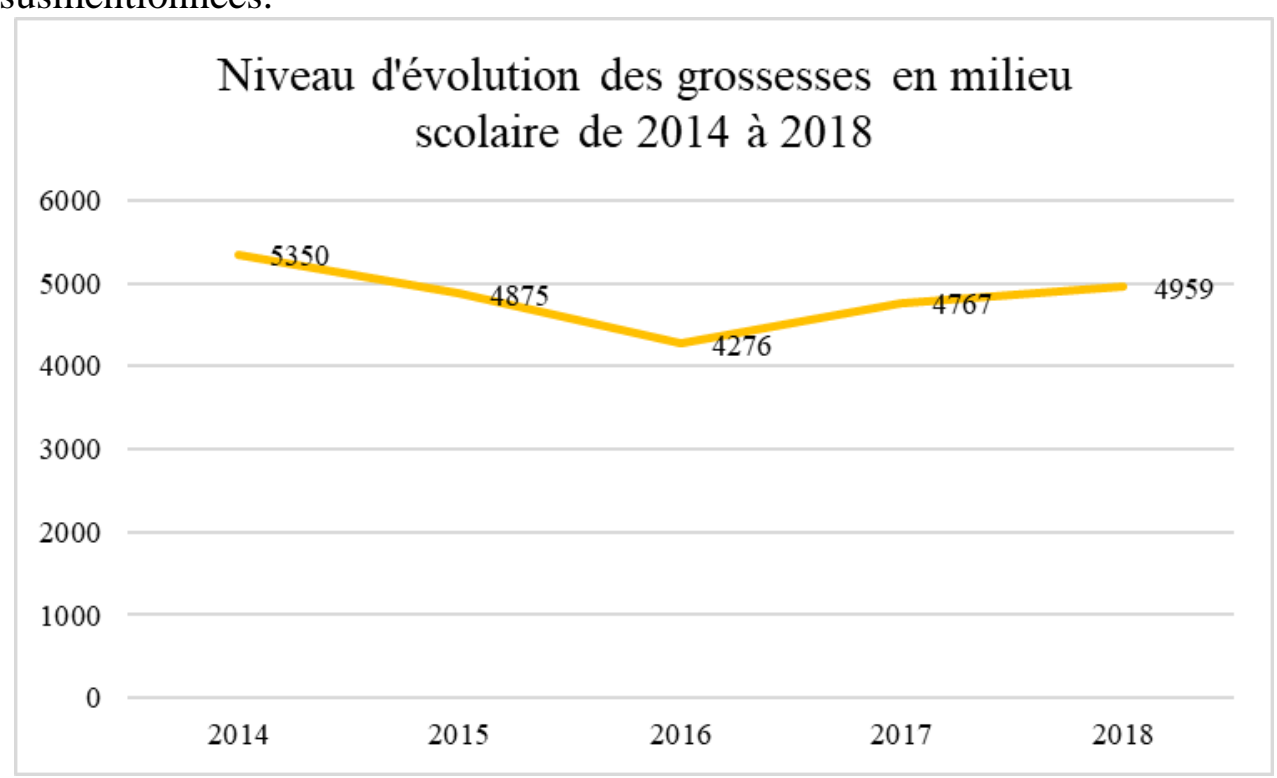

Figura 1.

Pour la seule année scolaire 2014, le taux des grossesses à l'école a connu un pic et cela malgré la mise en œuvre de la campagne de communication «Zéro grossesse à l'école ». De ce constat, il urge de préciser que la campagne "Zéro grossesse à l'école » lancée en 2014, n'a pas de fruits visibles. Du coup, une réorientation ou si l'on veut une recomposition des 
messages et de tout le processus de cette campagne de communication s'impose. La pratique des études de terrain nous enseigne que cette campagne a des insuffisances à plusieurs niveaux. À notre sens, c'est le contenu et les sens des messages véhiculés qui véritablement posent problème. Pour preuve, la première affiche de sensibilisation ayant attirée notre attention est la suivante :

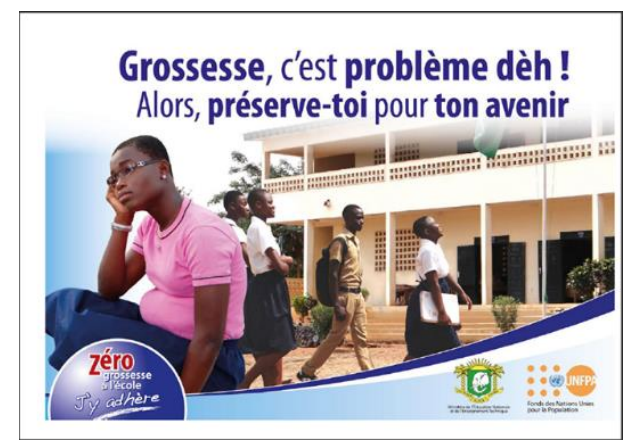

Figura 2.

En matière de communication pour le changement de comportements d'un individu, le principe de base est que la construction des messages doit être guidée par l'analyse (la recherche) et par la conception stratégique. Les messages doivent aussi avoir un potentiel émotionnel et artistique capable d'exercer une influence sur les gens ordinaires (Phyllis Tilson Piotrow et al., 1997).

En clair, une communication efficace intègre dans son élaboration les paramètres liés aux perceptions, attitudes, pratiques socioculturelles des populations bénéficiaires, de la cible. À la lumière de ces recommandations, l'affiche sus décrite, présente des incohérences. Lorsque nous l'avons montrée aux élèves, ils nous ont confié dans leur grande majorité ne pas percevoir le problème que pourrait constituer la grossesse à l'école.

«Des filles enceintes viennent à l'école, participent aux différents cours et réussissent à obtenir la moyenne pour aller en classe supérieur et/ou réussissent à un examen. », nous ont confié les enquêtés.

La deuxième affiche ayant retenue également notre attention comme présentant des insuffisances est celle-ci: 


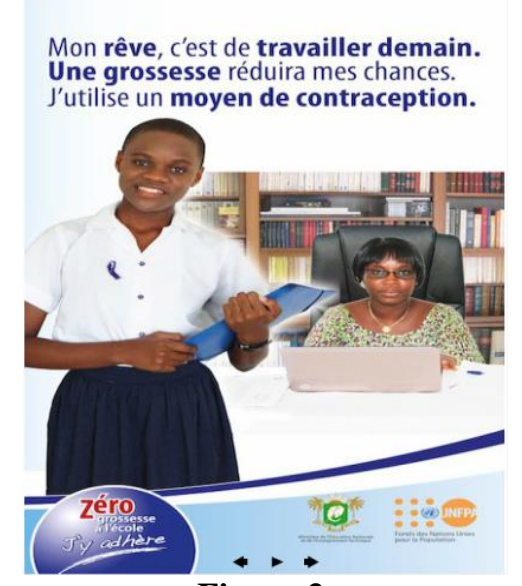

Figura 3.

Cette affiche occasionne des difficultés de compréhension chez les élèves rencontrés et au-delà chez les adultes. Les enquêtés nous ont dit ne pas connaitre les éléments constituant le moyen de contraception dont parlent les concepteurs de cette affiche. Quelle signification a le groupe de mots « moyen de contraception »? Nous ont-ils posé. Chez les adultes, la gêne est d'autant plus grande. Ces derniers estiment que cette affiche invite, tout comme les autres, les jeunes filles à avoir des rapports sexuels très tôt (entre 14 et 15 ans). À son jeune âge, la fille figurant sur l'affiche ne devrait pas songer de sitôt aux moyens de contraception pour éviter les grossesses à l'école. C'est la preuve que cette publicité est un échec. L'analyse de cette affiche laisse apparaitre une invitation implicite adressée aux adolescent(e)s à avoir des relations sexuelles très tôt et pour éviter les grossesses, leur salut viendrait de l'utilisation des moyens de contraception. Pour rappel, les affiches et/ou messages de sensibilisation invitant les jeunes à l'abstinence n'ont pas porté des fruits probants de sorte qu'aujourd'hui, les concepteurs des affiches et/ou messages de sensibilisation décident de prôner auprès de la jeunesse l'usage des moyens de contraception. Cela apparait ici comme une caution pour le vagabondage sexuel des élèves et partant de la jeunesse. De ce constat, des inquiétudes apparaissent: "Qu'en sera-t-il lorsque la campagne pour l'utilisation des moyens de contraception dans la sexualité chez les jeunes ne marchera pas ? Serons-nous amenés à leur montrer des publicités qui prônent comment avorter sans risque ? Va-t-on faire des campagnes obligatoires pour injecter des contraceptifs à longue durée aux élèves filles ? » Pour nous, les messages de sensibilisation contre les grossesses à l'école ne tablent pas sur les perceptions, attitudes et pratiques socioculturelles des élèves.

L'agence ivoirienne de marketing sociale (AIMAS), une ONG œuvrant aux côtés des ministères de l'Éducation nationale et de la santé 
publique dans la lutte contre les grossesses à l'école, a conçu des affiches dont une a également retenu notre attention.

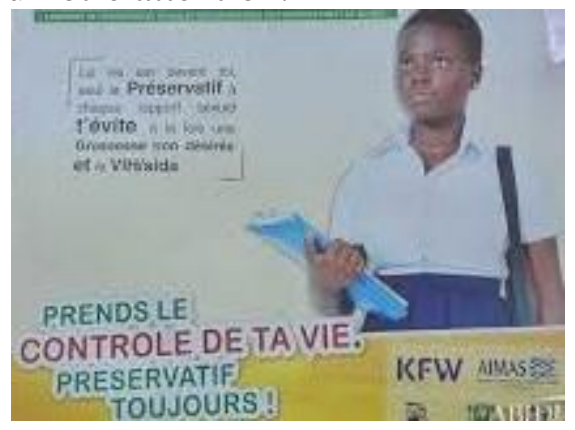

Figura 4.

Il est inscrit sur cette affiche en haut à gauche le message ci-après :

«La vie est devant toi, seul le Préservatif à chaque rapport sexuel t'évite à la fois une grossesse non désirée et le VIH/Sida. Prends le contrôle de ta vie. Préservatif toujours! ».

L'analyse de toutes ces affiches laissent apparaitre le même message à savoir «ayez des rapports sexuels et à chaque rapport, utilisez le préservatif $\gg$. Des contradictions apparaissent dans des messages de lutte contre les grossesses à l'école des jeunes filles : «Abstiens-toi de tout rapport sexuel en donnant la priorité à tes études » et «utilise aussi les moyens de contraception pour éviter les grossesses » apparaissent le plus souvent dans un même message. Pour les élèves que nous avons rencontrés, le message ou information à eux porter est clair et ne souffre d'aucune ambiguïté : «la jeune fille élève et/ou le jeune garçon élève peut avoir des rapports sexuels et pour éviter les grossesses chez les jeunes filles, elles doivent faire usage des moyens de contraception ce qui ne gêne ou ne perturbera en rien ses études ». Nous pensons qu'à travers cette insistance sur l'usage des moyens de contraception, les initiateurs de la lutte sont conscients de ce que les élèves ne peuvent résister à la tentation du sexe, à la pratique du sexe.

Par ailleurs, dans la conception des messages de lutte contre les grossesses chez les jeunes filles, l'état et ses partenaires ont décidé de produire des affiches adressées aux adultes qu'ils regroupent sous le vocable «éducateurs ». 


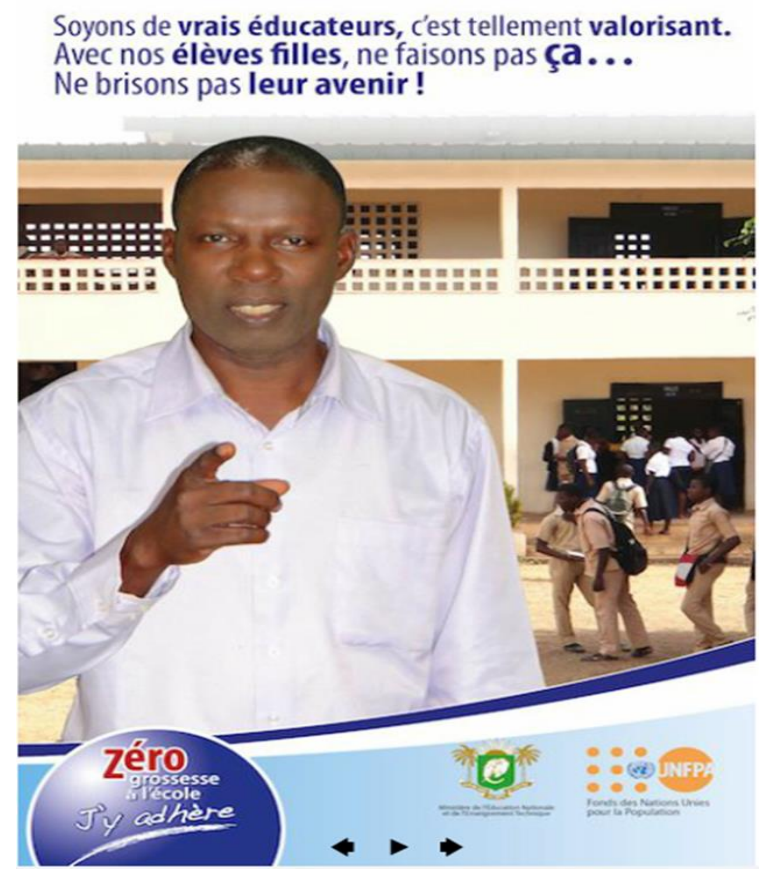

Figura 5.

Analyse faite de cette affiche, elle traduit une sorte d'ambigüité. Le message évoqué contient des imprécisions au niveau des termes suivants «vrais éducateurs»; «valorisant» et «ça ». Dans nos établissements d'enseignement secondaire, nous avons des personnes exerçant le métier de « éducateur » : est-ce ces derniers qui doivent s'interdire de faire «ça » avec leurs élèves filles ? Que renferme le terme «ça» (diminutif de cela) ? Ces questions traduisent bien les imprécisions contenues dans certains termes du message à l'endroit de l'adulte. Le terme «éducateur » désigne la fonction qu'exerce une personne s'occupant de veiller quotidiennement à la bonne conduite et l'application des règles, dans les établissements scolaires, par les élèves. Du coup, il diffère de «enseignant » et «personnel administratif et technique ».

Par ailleurs, cette affiche légitime les rapports sexuels d'une part entre les élèves des deux sexes et d'autre part, elle proscrit aux « éducateurs » d'en faire de même : c'est-à-dire avoir des rapports sexuels avec leurs élèves filles. Selon une étude diligentée par le ministère de l'éducation nationale, les résultats ont montré que les personnes qui mettent enceinte les élèves filles, sont de loin les personnes exerçant de petites activités autour de l'école (gérants de cabines téléphoniques, les mécaniciens, etc.). À côté de ces derniers figurent les élèves garçons et après le personnel d'éducation. Élaborer des messages d'interdiction de rapports sexuels avec les élèves filles à 
l'endroit du personnel d'éducation uniquement tout en laissant de côté les personnes pratiquant les petits métiers autour des écoles et les élèves garçons et l'avidité financière (le goût prononcé pour le matériel) des élèves filles, nous apparait plus ou moins inopportun.

Les affiches et messages élaborés dans le cadre de la campagne de communication dénommée «zéro grossesse à l'école» renferment les croyances de leurs concepteurs ou énonciateurs, ce qui entrent du coup en conflit avec celles des « bénéficiaires » réels des actions.

\section{Discussion}

\subsection{Faiblesses de la communication de la société ivoirienne en matière de sexe}

Les incohérences relevées dans les affiches susmentionnées résultent aussi de l'échec de la société ivoirienne actuelle. L'on ne peut pas exhorter les jeunes élèves à pratiquer l'abstinence sexuelle et autoriser, d'une part, la diffusion, sur les chaines de la télévision ivoirienne et cryptée à longueur de journée des films où les gens passent leur temps à s'amouracher; où ce sont les gens les plus dépravés qui sont les plus cools; où il y a toujours un charmant couple hétérosexuel et/ou homosexuel; où il $\mathrm{y}$ a des filles et des garçons presque nus. D'autre part, l'on n'est pas en mesure d'interdire formellement par exemple : l'accès aux bars et aux boîtes de nuit aux mineurs ; la vente et/ou l'exposition de CD (compact disc), journaux et revues pornographiques dans la rue.

Pour venir à bout de ce problème, les parents et gouvernants se rabattent sur l'école. En d'autres termes, l'école est tenue soit responsable de l'éducation des enfants, soit du manque d'éducation constatée chez les élèves. Or, l'école de nos jours a perdu une bonne partie de ses attributs qui lui permettait d'avoir une véritable autorité sur les élèves : l'enseignant a une parodie d'autorité, un élément du décor, un épouvantail qui ne fait même plus peur dans la mesure où, celui-là n'a pas le droit de mettre dehors, de donner zéro à l'élève turbulent et perturbateur voire même de le gronder car il peut le traumatiser. Dans une telle configuration, les élèves (filles et garçons) se permettent toute sorte de comportements : ils portent, regardent ce qu'ils veulent et entrent au cours et à la maison à l'heure qu'ils souhaitent; ils n'engagent aucune discussion sur la sexualité et relation de confiance avec la communauté. En clair, ils apparaissent dans ces circonstances comme « de la volaille élevée en divagation ». Le mal est donc profond. Le changement de messages peut s'avérer payant pendant un moment, cependant si les causes endogènes ne sont pas mises à jour et traitées, le problème continuera, demeurera. 


\subsection{Comment sensibiliser au changement de comportement efficacement et durablement les élèves ?}

Les défis majeurs à relever dans les interventions en communication pour le changement de comportement, demeurent ceux de l'efficacité de l'action communicationnelle, de la prise de décision et du passage de l'intention d'agir à l'action concrète elle-même. Pour ce faire et à l'instar de Bahi (2002), nous estimons qu'il faut, dans une communication visant l'adoption de comportements nouveaux et de leur mise en œuvre, au préalable que les concepteurs disposent d'éléments nombreux sur la situation, les habitudes, les relations, le projet des enfants, et bien d'autres variables en rapport avec la cible.

Pour faire simple, la communication, en plus de mettre l'accent sur le message à véhiculer à la cible, doit tenir également compte du plan média ou moyen à utiliser pour établir le contact avec la cible (Bahi, 2002).

En Côte d'Ivoire, le drame réside dans le fait que dans la mise en œuvre d'une campagne de sensibilisation, les concepteurs ou initiateurs calquent ce qui s'est déjà déroulé ailleurs. C'est-à-dire que l'on assiste à une reproduction ou reconduction servile de la campagne produite dans un pays autre que celui où sa mise en œuvre est annoncée. Dans ces circonstances, ladite campagne ne pourra être efficace et ainsi elle ne parvient pas à corriger le problème constaté. Il convient alors de mettre en place une politique de communication qui tient compte des réalités et spécificités de l'endroit et de la cible visés.

Enfin, la communication a amorcé doit s'inscrire dans le sens d'une perspective participative et inclusive. Au-delà même du changement lexical, ce processus révèle de manière constante un renouvellement conceptuel et méthodologique.

Dans la pratique et dans le cadre de la campagne de sensibilisation «Zéro grossesse à l'école » initiée par le ministère de l'éducation nationale et ses partenaires, la stratégie de communication proprement dite doit : dans un premier temps hiérarchiser les objectifs. C'est-à-dire définir des objectifs (principaux et opérationnels) précis. Ensuite, il importe de connaitre et déterminer la véritable cible des actions (la cible doit être hiérarchisée à savoir la cible principale, la cible secondaire et la cible tertiaire). Après la connaissance et la délimitation de la cible, vient le moment d'élaborer des messages. Ces derniers doivent être d'une précision sans faille. Pour chaque cible il y'a lieu d'élaborer des messages spécifiques en fonction du niveau ou degré de compréhension ou de connaissances de la cible. Enfin, il convient de planifier ou réfléchir au choix des supports ou moyens de diffusion desdits messages. Les canaux à utiliser doivent prendre en compte les moyens traditionnels de communication (médias de masses : télévision, radio, affiche ; le corps à corps : la proximité, etc.) et les nouveaux outils de communication tels que le téléphone mobile, les réseaux sociaux, l'internet, etc. 
En dépit de toutes ces actions, il est indispensable voire impérieux de marquer un relâchement plus ou moins long pour faire un suivi-évaluation des actions sur le terrain en vue d'un recadrage éventuel et nécessaire de sorte à atteindre l'objectif escompté, fixé ou à atteindre.

\section{Conclusion}

La recrudescence des grossesses en milieu scolaire constitue à en point douter un problème de développement en ce vingt-unième siècle. De ce fait, le développement et l'épanouissement de l'élève fille se trouve compromis et partant celui de toute la société ivoirienne. Les statistiques alarmants dans ce domaine, ont sucité cette étude qui a permis de mettre à jour les disfonctionnements, les incohérences et les insuffisances dans lesdits affiches et messages issus de la campagne "Zéro grossesse à l'école». Les contenus des différents messages et images que nous avons soumis au jugement ou l'appréciation des élèves issus de toute la Côte d'Ivoire paraissent inadaptés. Dans la mesure où les référents des termes utilisés dans les écritures prêtent à confusion dans l'esprit des adolescent(e)s. les initiateurs de cette campagne de communication n'intègrent pas dans leurs messages, les éléments et variables en rapport avec les adolescent(e)s notamment les savoirs, habitudes, relations, projets et fonctionnement de cette catégorie de la population et leur rapport avec les enseignants, les parents.

Il est impératif de se situer en rupture par rapport à l'approche qui veut que l'on expose, voire autorise à la jeune fille élève à faire usage des moyens de contraception dès l'adolescence ; qu'on lui demande de s'abstenir de tous rapports sexuels et au même moment qu'on lui dise qu'elle peut recourir aussi aux moyens de contraception pour éviter les grossesses. Cette ambigüité dans les messages n'est-elle pas à la base de la montée plus ou moins fulgurante du taux de grossesse à l'école d'une année à une autre ? Dans un tel processus complexe d'idéation, il est d'autant plus important que la communication interpersonnelle puisse occuper une place de choix. La promotion des nouveaux modèles comportementaux, pour qu'elle fasse sens pour les élèves (filles et garçons), doit être construite dans un paradigme, alternatif à celui du modèle de la convergence dont certains chercheurs en font l'éloge.

Cet article postule l'idée selon laquelle une communication pour le changement de comportement chez une cible donnée doit examiner, au premier abord, quel sentiment ou quelle résonance les idées nouvelles construisent dans la conscience de celle-là et, au second abord, si le sens construit de ces innovations correspond, à des changements de normes sociales, dans la conscience des élèves, de la cible. Ainsi, comme le stipule Miège (2004) « ... le devenir des techniques de l'information et de la communication, nécessite cependant de s'appuyer sur une théorie de l'action 
renouvelée et adaptée aux pratiques émergeantes ; ... seule une pensée de la produ-action ... répond à de telles conditions ».

\section{References:}

1. Abouo Joseph (2017). «ces cas de grossesses en milieu scolaire qui prennent une ampleur inquiétante, quelles solutions idoines? », publie le dimanche 18 mai 2014 et consulte le 19 mai.

2. Aubin Claire, Jourdain Menninger Daniele \& Laurent Chambaud (2009). La prévention des grossesses non désirées : information, éducation et communication, Inspection générale des affaires sociales, rapport $\mathrm{n}^{\circ} \mathrm{rm}$ 2009-118a, République Française

3. Bahi, A. (2002). «opinions et attitudes des hommes face aux méthodes contraceptives modernes », in kasabyakasa $n^{\circ} 3$, revue ivoirienne d'anthropologie et de sociologie, Abidjan, EDUCI

4. Bourdieu Pierre (2000). esquisse d'une théorie de la pratique, Paris, Seuil

5. Dagnogo Gnere Blama. Les grossesses en milieu scolaire au prisme de la communication pour le changement de comportement : l'exemple du lycée moderne de tengrela en Côte d'Ivoire, département de sociologie, université Alassane Ouattara (Côte d'Ivoire)

6. Goutille Fabienne (2009). Connaissances, attitudes et pratiques dans l'éducation aux risques : mettre en æuvve les études cap, Édition Handicap international-pole publications professionnelles, Catherine Clavel, Catherine Dixon.

7. James, O., Prochaska, C., Diclemente, C. (1982). Thérapie transthéorique : vers un modèle de changement plus intégratif, Psychothérapie: théorie, recherche et pratique.

8. Louis Dubrisay \& Cyrille Jeannin (1946). Précis d'accouchement, 11e Éditions, Paris, Libraire Lamarre.

9. Miège Bernard (2004). L'information - communication, objet de connaissance, 2e édition, Bruxelles, de boeck, PUF.

10. Phyllis Tilson Piotrow, Lawrence Kincaid, Jose Grimon, \& Everett Rogers (1997). Communication sanitaire : leçons de la planification familiale et de la santé génésique Westport Conn, Praeger.

11. Regouby Christian (1988). La communication globale Comment construire le capital image de l'entreprise, les Éditions d'organisation, Paris.

12. Rodriguez-Tome, H., Jackson, S., \& Bariaud, F. (1997). Regards actuels sur l'adolescence, Paris, P.U.F.

13. Syddick Aboubacar Al (2017). «Lutte contre les grossesses en milieu scolaire/Dr Ouattara Joséphine : "la guerre est désormais engagée contre ce fléau », infoivoire.net, consulte le 19 mai. 